\title{
Simultaneous Doping Analysis of Main Urinary Metabolites of Anabolic Steroids in Horse by Ion-trap Gas Chromatography-Tandem Mass Spectrometry
}

\author{
Masayuki Yamada, ${ }^{* \dagger}$ Sugako Aramaki, ${ }^{*}$ Masahiko Kurosawa,* Isao KiJIMA-SudA,* \\ Koichi SAITO, ** and Hiroyuki NAKAZAWA** \\ *Laboratory of Racing Chemistry, 1731-2 Tsuruta, Utsunomiya, Tochigi 320-0851, Japan \\ **Department of Analytical Chemistry, Hoshi University, 2-4-41 Ebara, Tokyo 142-8501, Japan
}

\begin{abstract}
The use of anabolic steroids in racehorses is strictly regulated. We have developed a method for the simultaneous analysis of 11 anabolic steroids: fluoxymesterone, $17 \alpha$-methyltestosterone, mestanolone, methandienone, methandriol, oxymetholone, boldenone, furazabol, methenolone, nandrolone, and stanozolol, for possible application to a doping test in racehorses. We selected 15 kinds of target substances for a doping test from the main metabolites of these anabolic steroids, and established a method for simultaneous analysis. Urine was hydrolyzed and subjected to solid-phase extraction. Then, the residue from the extracts was derivatized by trimethylsilylation. The derivatized samples were subjected to ion-trap gas chromatography-tandem mass spectrometry, and their mass chromatograms and product ion spectra were obtained. The limit of detection of the target substances was $5-50 \mathrm{ng} / \mathrm{mL}$, and the mean recovery and coefficient of variation were $71.3-104.8 \%$ and $1.1-9.5 \%$, respectively.
\end{abstract}

(Received June 10, 2008; Accepted July 22, 2008; Published September 10, 2008)

Anabolic steroids are used to treat wasting disease or osteoporosis, and to increase muscle growth and appetite in livestock. However, since they can increase muscle mass and physical strength, they are abused for the purpose of enhancing physical performance in racehorses and athletes. Accordingly, their use is now forbidden in racehorses and athletes, and doping test laboratories have been requested to develop methods for the detection of possibly misused anabolic steroids. We previously studied six anabolic steroids: fluoxymesterone (FOM), $17 \alpha$ methyltestosterone (MTS), mestanolone (MSL), methandienone (MDI), methandriol (MDO), and oxymetholone (OXM), ${ }^{1-3}$ and determined their main urinary metabolites on the basis of GC/MS data. We have also synthesized those metabolites, and used them as reference standards for identification. In addition, there are several reports of boldenone (BLD), furazabol (FRZ), methenolone (MNL), nandrolone (NAD), and stanozolol (STZ) and their metabolites. ${ }^{4-11}$ In the doping test for racehorses, the main metabolites of abused doping drugs are generally chosen as target substances, and their simultaneous analysis is carried out. However, although a simultaneous analysis of the parent steroids of the above-mentioned anabolic steroids has already been reported, ${ }^{6}$ as far as we know, simultaneous analysis of the main metabolites of the 11 anabolic steroids in horse urine has not been reported, and we could not find any confirmatory analysis of their metabolites for identification.

In this study, we selected urinary metabolites from 11 anabolic steroids (BLD, FOM, FRZ, MSL, MDI, MDO, MNL, MTS, NAD, OXM, and STZ) as target substances for the doping test in racehorses, and established a method for the simultaneous analysis of 15 target substances using ion-trap gas chromatography-tandem mass spectrometry (GC/MS/MS).

$\dagger$ To whom correspondence should be addressed.

E-mail: m-yamada@lrc.or.jp

\section{Materials and Methods}

\section{Steroids}

MTS, MSL, NAD, and STZ were obtained from SigmaAldrich Japan (Tokyo, Japan); MDI, from Gedeon Richter (Budapest, Hungary); MDO, from Diosynth (Oss, The Netherlands); OXM, from Edmond Pharma (Paderno Dugnano, MI, Italy); BLD and MNL, from Steraloids (Newport, RI); FOM, from Kanto Kagaku Reagent Division (Tokyo, Japan); and Deca Duramin ${ }^{\circledR}$ (NAD decanoate), from Fuji Pharmaceutical (Tokyo, Japan). $5 \alpha$-Estran-3 $\beta, 17 \alpha$-diol, the main metabolite of NAD, was obtained from Steraloids. $16 \alpha$-Hydroxyfurazabol was synthesized in our laboratory according to a report of Takegoshi et al. ${ }^{7} \quad 16 \alpha$-Hydroxystanozolol and $16 \beta$-hydroxystanozolol were synthesized in our laboratory according to a report of Schänzer et al. ${ }^{12} \quad 17 \alpha$-Methyl-5 $\alpha$-androstan$3 \beta, 16 \beta, 17 \beta$-triol, a metabolite common to MTS, MSL, MDO, MDI, and OXM, and $17 \alpha$-methyl-5 $\beta$-androstan- $3 \alpha, 16 \beta, 17 \beta$ triol, a metabolite common to MDO, MDI, and MTS, were synthesized in our laboratory according to our previous report. ${ }^{1}$ $9 \alpha$-Fluoro-17,17-dimethyl-19-norandrostane-4,13-diene-11 $\beta$-ol3 -one, the main metabolite of FOM, was synthesized in our laboratory according to our previous report. ${ }^{3}\left[16,16,17-{ }^{2} \mathrm{H}_{3}\right]-5 \alpha-$ Androstane- $3 \alpha, 17 \beta$-diol $\left({ }^{2} \mathrm{H}_{3}\right.$-AND), which was used as an internal standard (IS), was synthesized in our laboratory according to a report of Dehennin et al. ${ }^{13}$

\section{Chemicals and reagents}

$\mathrm{N}$-Methyl- $\mathrm{N}$-(trimethylsilyl)trifluoroacetamide (MSTFA) was purchased from GL Sciences (Tokyo, Japan). A Sep-Pak Plus $\mathrm{C}_{18} 360 \mathrm{mg} / \mathrm{cartridge}$ (Nihon Waters, Tokyo, Japan) was used as an SPE column, and a $1 \mathrm{M}$ hydrogen chloride-methanol solution was obtained from Kokusan Kagaku (Tokyo, Japan). All other 
reagents and solvents were of analytical or HPLC grade (purchased from Wako Pure Chemical Industries, Tokyo, Japan or Kanto Kagaku Reagent Division, Japan).

\section{Urine samples}

We used the urine samples of post-race female or gelding thoroughbreds submitted for routine doping tests as authentic urine samples. In order to obtain urine samples after NAD administration, we used three male experimental thoroughbreds housed at the Equine Research Institute, Japan Racing Association. NAD decanoate was administered intramuscularly to each horse at $0.8 \mathrm{mg} / \mathrm{kg}$. Urine samples were collected prior to NAD decanoate administration, and post NAD decanoate administration from each horse, and stored at temperatures below $-40^{\circ} \mathrm{C}$ prior to analysis. All experimental procedures in this study were approved by the Animal Care Committee of the Equine Research Institute, Japan Racing Association.

\section{Selection of target substances}

Regarding the target substance for the doping test, we selected known main metabolites that were detected after anabolic steroid use. The structures of the parent steroids and the target substances are shown in Fig. 1.

Boldenone (BLD), methenolone ( $M N L)$. According to Dumasia et al., the parent steroid, itself, and the 17-epimer of sulfate and glucuronide conjugates were detected as main urinary metabolites of BLD in horse. ${ }^{4,5}$ On the other hand, the urinary metabolites of MNL in horse were reported by Yu et al.; namely, 17-epi-methenolone and the parent steroid were detected as glucuronide conjugates. ${ }^{6}$ Therefore, the target substances for simultaneous analysis used to determine BLD and MNL abuse were the parent steroids, themselves, because the acquisition of authentic reference standards is easy.

Fluoxymesterone (FOM). Prior to this study, we investigated FOM metabolism, and confirmed that $9 \alpha$-fluoro-17,17dimethyl-19-norandrostane-4,13-diene-11 $\beta$-ol-3-one (FDN) was the main metabolite of FOM in horse urine, the same as in humans. ${ }^{3}$ A small amount of the parent steroid was detected in horse urine after FOM administration. Therefore, the target substances for simultaneous analysis to determine FOM abuse were FOM and FDN.

Furazabol (FRZ), stanozolol (STZ). The main metabolite of FRZ in rat or human was reported to be the 16-hydroxylated product of FRZ by Takegoshi et al. and Gradeen et al. ${ }^{7,8}$ However, there is no report on the main metabolites in horse. On the other hand, McKinney et al. reported that the main urinary metabolite of STZ in horse is the 16-hydroxylated product of STZ. ${ }^{9}$ We synthesized reference standards of $16 \alpha$-hydroxyfurazabol, $16 \alpha$-hydroxystanozolol, and $16 \beta$ hydroxystanozolol, and identified each metabolite in our laboratory, but did not publish the results. As results, the main metabolites of FRZ and STZ were $16 \alpha$-hydroxyfurazabol (HFR) and 16 $\alpha$-hydroxystanozolol (HST), respectively. In addition, small amounts of the parent steroids were detected. Therefore, FRZ, HFR, STZ, and HST were selected as target substances.

Methandienone (MDI), methandriol (MDO), $17 \alpha$ methyltestosterone (MTS), mestanolone (MSL), oxymetholone $(O X M)$. We previously investigated the metabolism of MDI, MDO, MTS, MSL, and OXM, ${ }^{1,2}$ and confirmed that $17 \alpha$-methyl-5 $\alpha$-androstan- $3 \beta, 17 \beta$-diol, $17 \alpha$-hydroxymethyl-5 $\alpha$ androstan- $3 \beta, 17 \beta$-diol, $17 \alpha$-methyl- $5 \alpha$-androstan- $3 \beta, 16 \alpha, 17 \beta$ triol, and $17 \alpha$-methyl- $5 \alpha$-androstan- $3 \beta, 16 \beta, 17 \beta$-triol (MAT) were metabolites common to the five steroids. Further, the $5 \beta$-H structural metabolite $17 \alpha$-methyl-5 $\beta$-androstan- $3 \alpha, 16 \beta, 17 \beta$-triol (5 $\beta \mathrm{MAT})$ was detected in the urine of MDI-, MDO-, or MTS-
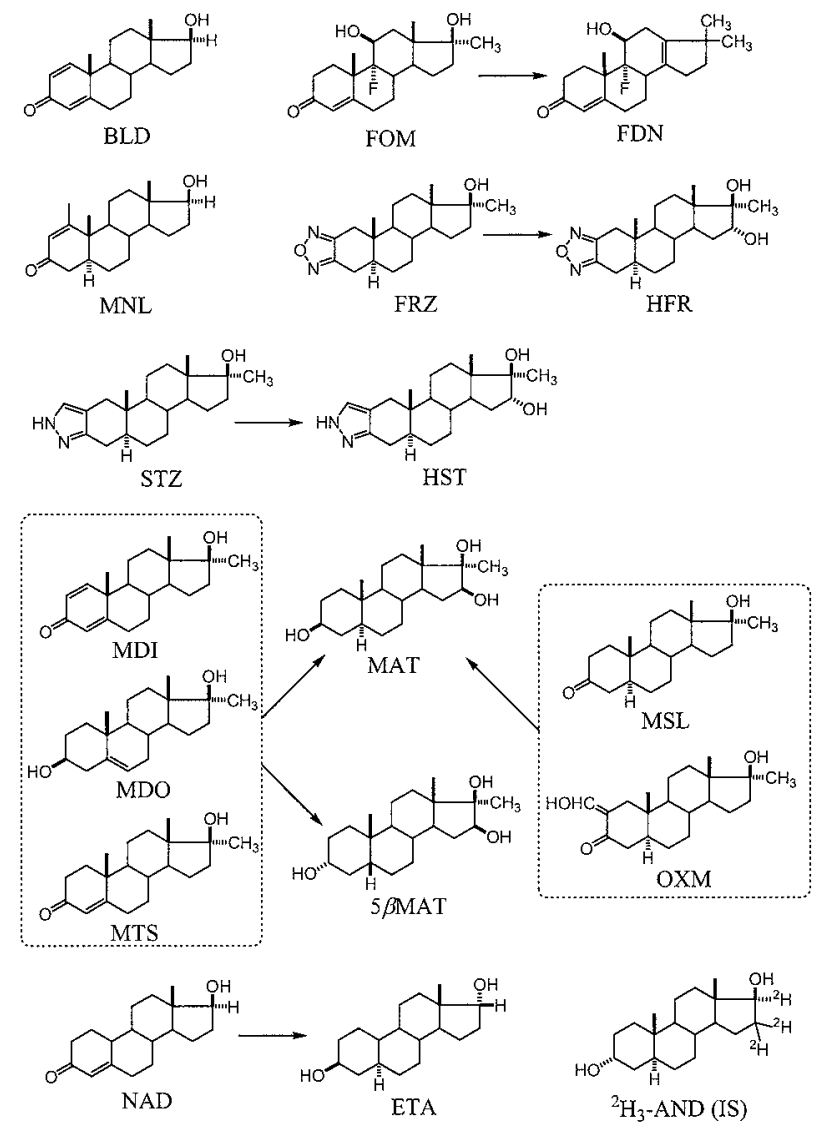

Fig. 1 Structures of anabolic steroids and their target substances for simultaneous analysis: BLD, boldenone; MNL, methenolone; FOM, fluoxymesterone; FDN, $9 \alpha$-fluoro-17,17-dimethyl-18-norandrostane4,13-dien-11 $\beta$-ol-3-one; FRZ, furazabol; HFR, $16 \alpha$-hydroxyfurazabol; STZ, stanozolol; HST, $16 \alpha$-hydroxystanozolol; MDI, methandienone; MDO, methandriol; MTS, $17 \alpha$-methyltestosterone; MSL, mestanolone; OXM, oxymetholone; MAT, $17 \alpha$-methyl-5 $\alpha$-androstan$3 \beta, 16 \beta, 17 \beta$-triol; $5 \beta$ MAT, $17 \alpha$-methyl-5 $\beta$-androstan- $3 \alpha, 16 \beta, 17 \beta$ triol; NAD, nandrolone; ETA, $5 \alpha$-estran- $3 \beta, 17 \alpha$-diol; ${ }^{2} \mathrm{H}_{3}$-AND, $\left[16,16,17-{ }^{2} \mathrm{H}_{3}\right]-5 \alpha$-androstane- $3 \alpha, 17 \beta$-diol.

administered horses, but not in MSL- or OXM-administered ones. In addition, small amounts of the parent steroids were detected in horse urine after MTS, MDO, and MDI administration. MSL was detected in the urine of OXMadministered horse despite the fact that it was hardly detected in the urine of MSL-administered horse. Among the metabolites mentioned above, we have reported that MAT is a useful screening target for a doping test of the five steroids in racehorses, and we selected it as the target substance for simultaneous analysis to determine MDI, MDO, MTS, MSL, or OXM abuse. In addition, MDI, MDO, MTS, MSL, and 5 $\beta$ MAT were included as target substances, because these would help us to narrow down the candidate administered steroid.

Nandrolone $(N A D)$. Urinary metabolites of NAD were investigated in detail by Houghton and Teale, who reported that NAD was mainly excreted as $5 \alpha$-estran- $3 \beta, 17 \alpha$-diol (ETA) sulfate and glucuronide in horse urine..$^{10,11}$ Therefore, ETA, the urinary main metabolite of NAD, was selected as the target substance in the simultaneous analysis to determine NAD abuse.

\section{Sample preparation}

Urine $(3 \mathrm{~mL})$ was adjusted to $\mathrm{pH} 5.0$ with $1 \mathrm{M}$ acetate buffer $(4.0 \mathrm{~mL})$ and ${ }^{2} \mathrm{H}_{3}$-AND (500 ng) was added as IS. The mixture 
Table 1 Partial mass fragments of target substances and selected precursor ions for GC/MS/MS

\begin{tabular}{llcr}
\hline Target substance & Characteristic ion in GC/MS scan data $(\mathrm{m} / z)$ & Precursor ion for GC/MS/MS $(\mathrm{m} / z)$ & Retention time/min \\
\hline BLD, bis-TMS & $73,191,206,229,299,325,340,415,430$ & $430\left(\mathrm{M}^{+}\right)$ & 8.11 \\
ETA, bis-TMS & $73,145,185,201,242,317,332,407,422$ & $407(\mathrm{M}-15)$ & 6.59 \\
FRZ, mono-TMS & $73,130,143,297,312,332,345,387,402$ & $387(\mathrm{M}-15)$ & 10.23 \\
HFR, bis-TMS & $143,218,231,283,311,332,400,475,490$ & $490\left(\mathrm{M}^{+}\right)$ & 11.55 \\
FOM, bis-TMS & $247,335,337,355,375,390,416,460,480$ & $480\left(\mathrm{M}^{+}\right)$ & 10.23 \\
FDN, bis-TMS & $73,193,208,247,267,337,357,372,462$ & $462\left(\mathrm{M}^{+}\right)$ & 7.47 \\
MDI, bis-TMS & $191,206,297,299,339,354,429,444$ & $444\left(\mathrm{M}^{+}\right)$ & 8.51 \\
MDO, bis-TMS & $73,197,211,227,253,268,343,358,433$ & $358\left(\mathrm{M}^{-} 90\right)$ & 8.33 \\
MSL, bis-TMS & $73,143,216,268,343,358,419,433,448$ & $448\left(\mathrm{M}^{+}\right)$ & 8.43 \\
MTS, bis-TMS & $213,251,301,314,341,356,431,446$ & $446\left(\mathrm{M}^{+}\right)$ & 8.57 \\
MAT, tris-TMS & $73,218,231,269,331,358,448,523,538$ & $538\left(\mathrm{M}^{+}\right)$ & 10.08 \\
5 $\beta$ MAT, tris-TMS & $73,218,231,269,331,358,448,523,538$ & $538\left(\mathrm{M}^{+}\right)$ & 9.32 \\
MNL, bis-TMS & $73,105,179,195,208,341,356,431,446$ & $446\left(\mathrm{M}^{+}\right)$ & 8.29 \\
STZ, bis-TMS & $73,143,168,342,367,381,382,457,472$ & $472\left(\mathrm{M}^{+}\right)$ & 11.21 \\
HST, tris-TMS & $218,231,328,353,380,381,470,545,560$ & $560\left(\mathrm{M}^{+}\right)$ & 12.35 \\
${ }^{2}{ }_{3}$-AND, bis-TMS & $73,131,215,244,259,334,349,424,439$ & $334\left(\mathrm{M}^{-}-105\right)$ & 7.23 \\
\hline
\end{tabular}

was centrifuged at $1663 \mathrm{~g}$ (radius of gyration, $16.5 \mathrm{~cm}$; rpm, 3000) for $10 \mathrm{~min}$, and loaded onto a Sep-Pak Plus $\mathrm{C}_{18}$ cartridge (pretreated with $5 \mathrm{~mL}$ of methanol and $5 \mathrm{~mL}$ of deionized water). The cartridge was rinsed with $5 \mathrm{~mL}$ of deionized water and $5 \mathrm{~mL}$ of $n$-hexane. Then, the cartridge was dried under a vacuum and eluted with $5 \mathrm{~mL}$ of ethyl acetate/methanol $(5: 1)$. The eluate was evaporated under nitrogen at temperatures below $40^{\circ} \mathrm{C}$ and the residue was dissolved in a $1 \mathrm{M}$ anhydrous hydrogen chloride-methanol solution $(0.5 \mathrm{~mL})$ and incubated for $10 \mathrm{~min}$ at $60^{\circ} \mathrm{C}$. Then, the residue was dissolved in diethyl ether $(5 \mathrm{~mL})$. Next, the solution was washed with $1.0 \mathrm{M}$ sodium hydroxide and $0.15 \mathrm{M}$ sodium chloride in water $(2 \mathrm{~mL})$. The solvents were evaporated under nitrogen at temperatures below $40^{\circ} \mathrm{C}$. After evaporation, the residue was dissolved in $50 \mu \mathrm{L}$ of $\mathrm{NH}_{4} \mathrm{I} /$ dithioerythritol/MSTFA $(2 \mathrm{mg} / 4 \mathrm{mg} / 1 \mathrm{~mL})$, and the reaction was allowed to proceed at $80^{\circ} \mathrm{C}$ for $30 \mathrm{~min}$. Finally, $2 \mu \mathrm{L}$ of this solution was injected into GC/MS/MS.

\section{GC/MS/MS analysis}

GC/MS/MS was performed on a gas chromatograph coupled with a GCQ ion-trap mass spectrometer and equipped with an A200S GC auto-sampler (Finnigan Thermo, CA). The fusedsilica capillary column used was DB-5ms (J \& W Scientific, $\mathrm{CA}$ ), having $15 \mathrm{~m} \times 0.25 \mathrm{~mm}$ i.d. and $0.25 \mu \mathrm{m}$ film thickness. Helium was used as the carrier gas $(50 \mathrm{~cm} / \mathrm{s})$, and the column oven temperature was programmed to increase from 150 to $320^{\circ} \mathrm{C}$ at $15^{\circ} \mathrm{C} / \mathrm{min}$, and maintained at $320^{\circ} \mathrm{C}$ for $1 \mathrm{~min}$. The injector temperature was set at $250^{\circ} \mathrm{C}$ and the MS transfer line temperature at $275^{\circ} \mathrm{C}$. The collision energy was $1.0 \mathrm{eV}$ and the excitation energy ( $q_{z}$ value) was 0.225 for all GC/MS/MS analyses.

\section{Results and Discussion}

\section{Study of a simultaneous analysis method}

Target substances exist in unconjugated free, glucuronide, and sulfate forms in horse urine, and therefore conjugate decomposition should be performed prior to simultaneous analysis of the target substances. Although enzyme hydrolysis is generally used, this method is not applicable to some anabolic steroids conjugated with sulfate. The decomposition of sulfates or glucuronides with methanolysis using anhydrous hydrogen

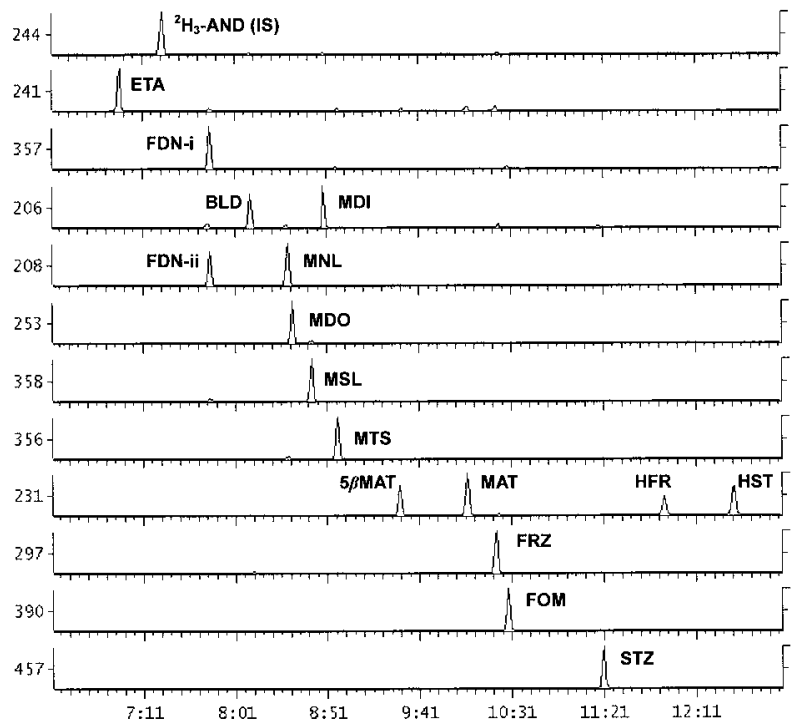

Fig. 2 Typical selected product-ion chromatograms of the trimethylsilyl derivatives of target substances from GC/MS/MS analysis: ${ }^{2} \mathrm{H}_{3}$-AND, bis-TMS (IS, $m / z, 334 \rightarrow 244$ ); ETA, bis-TMS $(\mathrm{m} / z 407 \rightarrow 241)$; FDN-i, bis-TMS $(\mathrm{m} / z 462 \rightarrow 357)$; FDN-ii, bisTMS $(\mathrm{m} / \mathrm{z} 462 \rightarrow 208)$; BLD, bis-TMS $(\mathrm{m} / \mathrm{z} 430 \rightarrow 206)$; MDI, bisTMS ( $m / z 444 \rightarrow 206)$; MNL, bis-TMS ( $m / z 446 \rightarrow 208)$; MDO, bisTMS $(m / z, 358 \rightarrow 253)$; MSL, bis-TMS $(m / z, 448 \rightarrow 358)$; MTS, bisTMS $(m / z 446 \rightarrow 356) ; 5 \beta$ MAT, tris-TMS $(m / z, 538 \rightarrow 231)$; MAT, tris-TMS $(m / z, 538 \rightarrow 231)$; HFR, bis-TMS $(m / z, 490 \rightarrow 231)$; HST, tris-TMS $(\mathrm{m} / \mathrm{z} 560 \rightarrow 231)$; FRZ, mono-TMS $(\mathrm{m} / \mathrm{z} 387 \rightarrow 297)$; FOM, bis-TMS $(\mathrm{m} / \mathrm{z} 480 \rightarrow 390)$; STZ, bis-TMS $(\mathrm{m} / \mathrm{z}, 472 \rightarrow 457)$.

chloride-methanol has been reported. ${ }^{14}$ Regarding derivatives of anabolic steroids and their metabolites, Stanley et al. reported the use of a mixed reagent of $\mathrm{NH}_{4} \mathrm{I}$, dithioerythritol, and MSTFA, and obtained good results. ${ }^{15}$ We adopted the mixed reagent for derivatives of target substances in this study. As a result, single trimethylsilylation products containing the keto or amino group were obtained, which enabled simultaneous analysis with high sensitivity and stability of most of the target substances.

In our simultaneous analysis, we employed GC/MS/MS, 


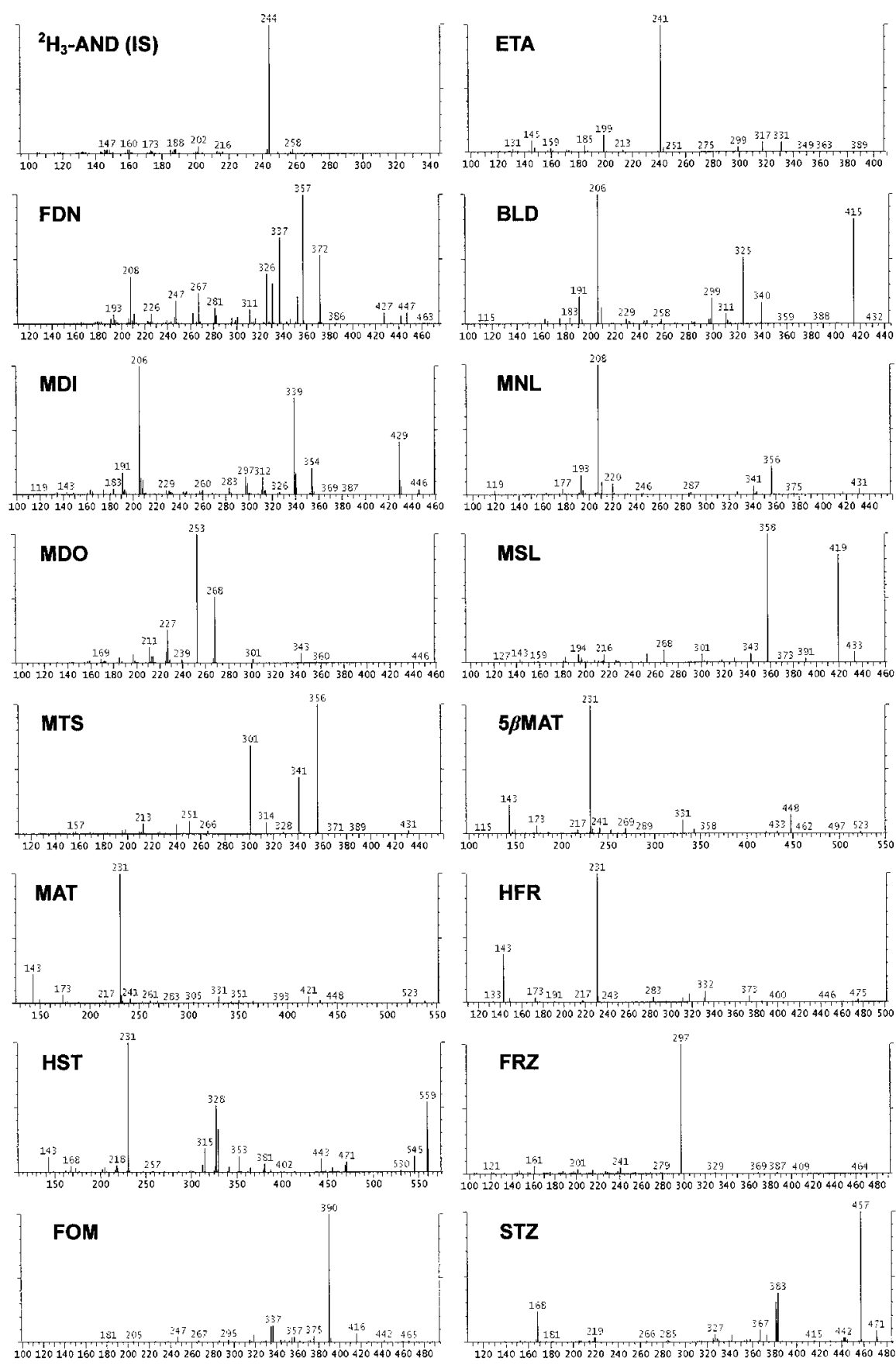

Fig. 3 Product ion spectra of the trimethylsilyl derivatives of target substances from GC/MS/MS analysis: ${ }^{2} \mathrm{H}_{3}$-AND, bis-TMS (IS); ETA, bis-TMS; FDN, bis-TMS; BLD, bis-TMS; MDI, bis-TMS; MNL, bis-TMS; MDO, bis-TMS; MSL, bis-TMS; MTS, bis-TMS; $5 \beta$ MAT, tris-TMS; MAT, trisTMS; HFR, bis-TMS; HST, tris-TMS; FRZ, mono-TMS; FOM, bis-TMS; STZ, bis-TMS.

which has high sensitivity and specificity. The simultaneous analysis of GC/MS/MS analysis is attained by changing the precursor ion according to the retention time of the target substance. Prior to GC/MS/MS analysis, GC/MS full-scan data of the target compound were obtained with GCQ, and the precursor ion of GC/MS/MS was selected based on the obtained mass spectrum data. The retention times, full-scan data, and the selected precursor ions with the target substances are given in Table 1. Although the molecular ion was selected as a precursor ion for most of the target substances, when the relative intensity of the molecular ion was weak, and sufficient sensitivity could not be obtained, the quasi-molecular ion was chosen. Typical selected product-ion chromatograms of the target substances from GC/MS/MS analysis are shown in Fig. 2, and their product ion spectra are shown in Fig. 3. The peak of each target substance was able to separate clearly in the product-ion chromatogram.

\section{Method validation}

Sensitivity. The limit of detection (LOD) in this simultaneous analysis is the minimum concentration that met the minimum criteria for identification provided by the Association of Official Racing Chemists. ${ }^{16}$ The criteria include the following: the retention time of the test sample is in agreement with that of the 
reference standard; the relative abundance (RA) of more than three characteristic ions is within the tolerance level; all ions appearing in the reference standard mass spectrum with an RA greater than $10 \%$ must also be present in the test sample mass spectrum; and any extraneous ions present should not exceed 20\% RA. As shown in Table 2, LOD of the target substances was $5-50 \mathrm{ng} / \mathrm{mL}$. Among the anabolic steroids investigated in this study, BLD is listed for performance specification in laboratories for doping control required by International Federation of Horseracing Authorities (IFHA), and the minimum urinary concentration for detecting exposure is $20 \mathrm{ng} / \mathrm{mL}$ in horse. In the simultaneous analysis employed in this study, LOD of BLD was lower than that specified by IFHA.

Specificity and reproducibility. To evaluate the specificity and reproducibility, the above-described method was assessed using different authentic urine samples and spiked urine samples with all of the target substances at the LOD concentrations listed in Table 2. Each urine sample was examined by replicate analysis $(n=10)$ on three different days. As a result, all control urine

Table 2 Limits of detection and recovery rates and their coefficient of variation of simultaneous analysis

\begin{tabular}{lcrc}
\hline \multirow{2}{*}{$\begin{array}{c}\text { Target } \\
\text { substance }\end{array}$} & \multirow{2}{*}{$\begin{array}{c}\text { LOD/ } \\
\mathrm{ng} \mathrm{mL}^{-1}\end{array}$} & \multicolumn{2}{c}{ Recovery rate, $\%$} \\
\cline { 3 - 4 } & & $50 \mathrm{ng} \mathrm{mL}^{-1}$ & $500 \mathrm{ng} \mathrm{mL}^{-1}$ \\
\hline BLD & 10 & $99.0 \pm 1.7$ & $93.8 \pm 4.5$ \\
ETA & 10 & $91.4 \pm 1.3$ & $95.1 \pm 2.2$ \\
FRZ & 20 & $79.2 \pm 2.2$ & $86.1 \pm 1.6$ \\
HFR & 20 & $75.3 \pm 6.8$ & $71.3 \pm 9.5$ \\
FOM & 10 & $82.6 \pm 6.1$ & $85.5 \pm 2.0$ \\
FDN & 5 & $73.5 \pm 3.9$ & $82.4 \pm 3.0$ \\
MDI & 10 & $95.7 \pm 4.2$ & $95.8 \pm 1.1$ \\
MDO & 5 & $75.3 \pm 2.1$ & $85.6 \pm 1.4$ \\
MSL & 10 & $104.8 \pm 5.7$ & $85.6 \pm 4.8$ \\
MTS & 5 & $80.8 \pm 1.6$ & $74.4 \pm 3.8$ \\
MAT & 20 & $88.8 \pm 3.7$ & $83.4 \pm 3.0$ \\
$5 \beta$ MAT & 20 & $73.0 \pm 7.9$ & $72.5 \pm 7.7$ \\
MNL & 10 & $78.2 \pm 2.2$ & $84.8 \pm 1.4$ \\
STZ & 20 & $78.4 \pm 1.7$ & $80.7 \pm 2.6$ \\
HST & 50 & $87.2 \pm 3.7$ & $91.1 \pm 1.9$ \\
${ }^{2} \mathrm{H}_{3}$-AND (IS) & & & $86.6 \pm 3.7$ \\
\hline
\end{tabular}

Mean $\pm \mathrm{CV}(\%), n=7$. samples gave negative results, while all of the spiked urine samples were positive.

Extraction recovery. The absolute mean recovery and the coefficient of variation (CV) for the target substances and the IS are given in Table 2 . The recovery rates exceeded $70 \%$, and CV was less than $10 \%$ for all of the target substances.

Nandrolone testing and application of method to administered samples

It is known that NAD is generated in the biosynthesis of estradiol from testosterone in male horse. ${ }^{10,11}$ Thus, its endogenous metabolites, which are not derived from NAD administration, are also excreted in urine. For this reason, NAD doping control in male horses has been conducted based on the threshold, namely, the concentration ratio of ETA to 5(10)estren-3 $\beta, 17 \alpha$-diol (ETE), an endogenous substance that is not derived from NAD administration. The threshold, ETA/ETE = $1 / 1$, is based on statistics of authentic horses provided by IFHA. Therefore, ETA, the urinary main metabolite of NAD, was selected as the target substance in a simultaneous analysis to determine NAD abuse. Although ETE was not selected as the target substance in this simultaneous analysis, when ETA is detected in male horse, it is necessary to confirm the ETA/ETE concentration ratio according to IFHA's ETA/ETE threshold.

For applying the method to administered samples, we administered NAD decanoate to male experimental thoroughbreds, and the collected urine was used for simultaneous analysis. The mass chromatogram and the product ion spectrum of urine sampled day 23 after NAD decanoate administration are shown in Fig. 4. The urine in this investigation was used to measure the concentration ratio of ETA/ETE already obtained in a previous study; namely, urine samples from day 1 or 2 to day 23 after administration had ETA/ETE $\geq 1 / 1 .{ }^{17}$ Although ETA should be detected from the urine greater than a threshold, this simultaneous analysis method was able to well detect ETA in these urine samples.

In human sports, although NAD and testosterone are prohibited, and a threshold has been adopted for their detection, measuring the $\delta^{13} \mathrm{C}$ value of the endogenous substance by gas chromatography/combustion/carbon isotope ratio mass spectrometry (GC/C/IRMS) is also recommended by World Anti-Doping Agency (WADA). ${ }^{18}$ On the other hand, we investigated the applicability of GC/C/IRMS to the NAD doping test in racehorse, and found that the measurement of $\delta^{13} \mathrm{C}$ values
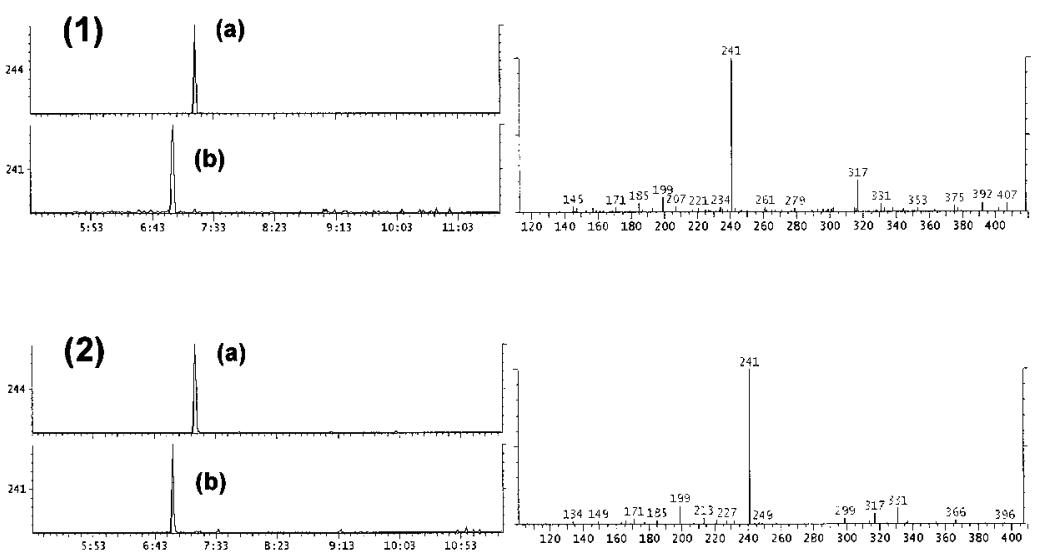

Fig. 4 Mass chromatograms and product ion spectra from GC/MS/MS analysis. (1) ETA reference standard; (2) urine sampled day 23 after NAD decanoate administration; peak a, ${ }^{2} \mathrm{H}_{3}$-AND (IS, $\mathrm{m} / \mathrm{z}$ $334 \rightarrow 244)$; peak b, ETA $(\mathrm{m} / \mathrm{z}, 407 \rightarrow 241)$. 
Table 3 Metabolites detected from urine of horse administered MTS, MSL, MDI, MDO, and OXM

\begin{tabular}{cl}
\hline Administered drug & \multicolumn{1}{c}{ Detected metabolite } \\
\hline MTS & MAT, $5 \beta$ MAT, MTS \\
MSL & MAT \\
MDI & MAT, $5 \beta$ MAT, MDI \\
MDO & MAT, $5 \beta$ MAT, MDO, MTS \\
OXM & MAT, MSL \\
\hline
\end{tabular}

with GC/C/IRMS enabled us to discriminate exogenous ETA derived from NAD-administered horse from endogenous ETA. ${ }^{17}$ Since there are reports of complex metabolic disorders in humans, ${ }^{19,20}$ the possibility of such disorders occurring in racehorses cannot be completely denied. Thus, GC/C/IRMS is a useful technique to complement the ETA/ETE concentration ratio in confirming NAD doping in racehorses.

17 $\alpha$-Methyltestosterone, mestanolone, methandienone, methandriol, and oxymetholone testing

Aside from MAT, we selected MTS, MDI, MDO, MSL, and $5 \beta$ MAT as target substances to determine MTS, MSL, MDI, MDO, and OXM doping in the confirmatory analysis. We did this because MAT is common to the above five anabolic steroids, and selected target substances would narrow down the candidate administered steroids. The detection results of these five steroids after administration are given in Table 3. It should be noted, however, that the metabolism of anabolic steroids is often dependent on the route of administration, and the metabolism of prodrugs may differ from the metabolism of their active forms. Therefore, identification of the abused drug should be conducted with care.

\section{Conclusion}

We examined a method for the simultaneous analysis of 15 kinds of metabolites from 11 anabolic steroids (BLD, FOM, FRZ, MDI, MDO, MNL, MTS, MSL, NAD, OXM, and STZ) for its possible application to a doping test for racehorses. Since the use of anabolic steroids should be strictly regulated in racehorses, we adopted GC/MS/MS for the simultaneous analysis, which has high sensitivity and specificity. Although methods for the simultaneous analysis of the parent steroids of the above-mentioned anabolic steroids have been reported, as far as we know, no method for the simultaneous analysis of the main urinary metabolites of the 11 anabolic steroids in horses has been reported. Therefore, our present findings are expected to contribute to improving the analysis of metabolites of anabolic steroids. This simultaneous analysis may have potential use for metabolites other than those in this study, and the results would contribute to an improvement of anabolic steroid analysis in racehorses.

\section{Acknowledgements}

The authors would like to thank the Equine Research Institute, Japan Racing Association, for assistance in performing nandrolone decanoate drug-administration experiments.

\section{References}

1. M. Yamada, S. Aramaki, T. Okayasu, T. Hosoe, M. Kurosawa, I. Kijima-Suda, K. Saito, and H. Nakazawa, J. Pharm. Biomed. Anal., 2007, 45, 125.

2. M. Yamada, S. Aramaki, M. Kurosawa, K. Saito, and H. Nakazawa, J. Anal. Toxicol., 2008, 32, 387.

3. M. Yamada, S. Aramaki, T. Hosoe, M. Kurosawa, I. KijimaSuda, K. Saito, and H. Nakazawa, Anal. Sci., 2008, 24, 911.

4. M. C. Dumasia, E. Houghton, C. V. Bradley, and D. H. Williams, Biomed. Mass Spectrom., 1983, 10, 434.

5. M. C. Dumasia, E. Houghton, and P. Tale, in Proceedings of the 6th International Conference of Racing Analyst and Veternarians, 1985, Hong Kong, 225 - 228.

6. N. H. Yu, E. N. Ho, D. K. Leung, and T. S. Wan, J. Pharm. Biomed. Anal., 2005, 37, 1031.

7. T. Takegoshi, H. Tachizawa, and G. Ota, Chem. Pharm. Bull. (Tokyo), 1972, 20, 1243.

8. C. Y. Gradeen, S. C. Chan, and P. S. Przybylski, J. Anal. Toxicol., 1990, 14, 120.

9. A. R. McKinney, C. J. Suann, A. J. Dunstan, S. L. Mulley, D. D. Ridley, and A. M. Stenhouse, J. Chromatogr., B, 2004, 811, 75 .

10. P. Teale and E. Houghton, Biol. Mass. Spectrom., 1991, 20 , 109.

11. E. Houghton, in Proceedings of the 9th International Conference of Racing Analyst and Veternarians, 1992, New Orleans, Louisiana, 3 - 16.

12. W. Schänzer, G. Opfermann, and M. Donike, J. Steroid Biochem., 1990, 36, 153.

13. L. Dehennin, A. Reiffsteck, and R. Scholler, Biomed. Mass Spectrom., 1980, 7, 493.

14. P. W. Tang and D. L. Crone, Anal. Biochem., 1989, 182, 289.

15. S. M. R Stanley, R. L. Brooksbank, and J. P. Rodgers, in Proceedings of the 10th International Conference of Racing Analyst and Veternarians, 1994, Stockholm, Sweden, 350 353.

16. P. V. Endoo and F. T. Delbeke, Chromatographia (Supplement), 2004, 59, S39.

17. M. Yamada, K. Kinoshita, M, Kurosawa, K. Saito, and H. Nakazawa, J. Pharm. Biomed. Anal., 2007, 45, 654.

18. WADA, Reporting the 2008 Prohibited List, World AntiDoping Agency, Montreal, Canada, http://www.wadaama.org/rtecontent/document/2008_List_En.pdf.

19. M. Ueki, Jpn. J. Toxicol. Environ. Health, 1998, 44, 75.

20. M. Ueki and M. Okano, Rapid. Commun. Mass Spectrom., 1999, 13, 2237. 